\title{
Muséologies
}

Les cahiers d'études supérieures

\section{Sylvie Lacerte, coordonnatrice de l'Alliance DOCAM, Fondation Daniel Langlois pour l'art, la science et la technologie}

\section{Marie-Hélène Foisy et Marie-Ève Courchesne}

Volume 2, numéro 1, octobre 2007

URI : https://id.erudit.org/iderudit/1033600ar

DOI : https://doi.org/10.7202/1033600ar

Aller au sommaire du numéro

Éditeur(s)

Association Québécoise de Promotion des Recherches Étudiantes en

Muséologie (AQPREM)

ISSN

1718-5181 (imprimé)

1929-7815 (numérique)

Découvrir la revue

Citer ce document

Foisy, M.-H. \& Courchesne, M.-È. (2007). Sylvie Lacerte, coordonnatrice de l'Alliance DOCAM, Fondation Daniel Langlois pour l'art, la science et la technologie. Muséologies, 2(1), 104-117. https://doi.org/10.7202/1033600ar d'utilisation que vous pouvez consulter en ligne. 


\section{Sylvie Lacerte,}

coordonnatrice de l'Alliance DOCAM

Fondation Daniel Langlois pour l'art,

la science et la technologie

[réalisée par Marie-Hélène Foisy et Marie-Ėve Courchesne le12 septembre 2007]

Sylvie Lacerte a complété un doctorat en études et pratiques des arts de l'UQÀM, ainsi qu'une maîtrise en museum studies à la New York University. Elle coordonne les travaux de l'Alliance Documentation et conservation du patrimoine des arts médiatiques (DOCAM) à la fondation Daniel Langlois pour l'art, la science et la technologie (FDL) depuis 2005. Pendant la session d'hiver 2007, elle était titulaire du séminaire DOCAM, offert à l'UOAMM [1], et sera à nouveau titulaire de ce séminaire au cours de la session d'hiver 2008 , cette fois à l'Université McGill. Ce séminaire s'inscrit dans le projet de l'Alliance DOCAM. Au printemps 2007, Sylvie Lacerte a publié un essai découlant de sa thèse, La médiation de l'art contemporain, aux Éditions d'art le Sabord. Cet ouvrage est en nomination pour le prix Spirale Éva-Le-Grand 2007

\section{[1]}

Ce Séminaire est offert depuis l'hiver 2006 en alternance par I'Université McGill et par l'Université du Québec à Montréal, pendant la durée du projet de recherche. 
M.-H. FOISY \& M.-È. COURCHESNE

$\mathbf{M}^{\mathrm{me}}$ Lacerte, s'il vous plaît décrivez-nous brièvement ce qu'est l'Alliance de recherche DOCAM.

SYLVIE LACERTE

Le projet Documentation et conservation du patrimoine des arts médiatiques (DOCAM) est une initiative de la fondation Daniel Langlois. Il s'agit d'un programme de recherche qui s'inscrit en continuité avec le projet $d u$ Réseau des médias variables ${ }^{[2]}$, réalisé conjointement parlafondation Langloiset lemusée Guggenheim de New York au debut des années 2000, dont les résultats ont été publiés en 2004 dans l'ouvrage bilingue La permanence par le changement. Le projet du Réseau des médias variables avait pour objectif de proposer de nouveaux outils de préservation des œuvres de la collection du Guggenheim, issues des mouvements de l'art conceptuel, minimaliste et des pratiques artistiques en vidéo. C'est en 2004 que Jean Gagnon et Alain Depocas de la FDL ont soumis ce vaste projet multidisciplinaire et interinstitutionnel, qu'est devenu DOCAM, au Conseil de Recherches en Sciences Humaines du Canada (CRSH), dans son programme Alliance de recherche université et communauté (ARUC) qui finance des projets échelonnés sur une durée de cinq ans. Il existe quatorze projets ARUC à travers le Canada, provenant de la même cohorte que celle de DOCAM

[2]

<http://www.variablemedianet/>. mais notre Alliance est la seule en arts, les autres se situant plutôt dans les domaines des sciences humaines et sociales. DOCAM est donc composé de partenaires universitaires tels l'UQÀM / Universitédu Québec à Montréal (École des arts visuels et médiatiques, CIAM/ Centre interuniversitaire des arts médiatiques et Faculté des sciences), l'Université de Montréal (École de bibliothéconomie et des sciences de l'information), l'Université McGill (Department of Art History and Communication Studies et École de musique Schulich) et Queen's University à Kingston en Ontario (Queen's Archives, programme de maitrise en restauration de l'art). Les partenaires de la communauté sont le Musée des beaux-arts du Canada (MBAC), le Musée d'art contemporain de Montréal, le Musée des beaux-arts de Montréal (MBAM) et le Centre Canadien d'Architecture (CCA). L'objectifprincipal de DOCAM est de déterminer comment préserver les œuvres en arts médiatiques et à composantes technologiques contenues dans les collections des musées. Il est intéressant de noter que des quinze projets ARUC, seulement trois sont gérés par un organisme de la communauté, dont DOCAM par l'intermédiaire de la fondation Daniel Langlois. C'est très important à retenir, car ce genre de collaboration entre les universités et la communauté permet au CRSH de transformer sa propre culture de financement de la recherche hors université. 
Cela explique par ailleurs pourquoi, dans les premiers mois du projet en 2005, nous avons passé un bon moment à travailler àl'harmonisation des méthodologies de recherches entre les universités et les musées... à nous apprivoiser en quelque sorte. C'était un elément crucial puisque les musées ont une vision de la recherche différente de celle des universités. En général, les musées apprêhendent la recherche par le biais d'une approche empirique, avec l'exposition comme résultat ultime, tandis que la tradition de la recherche universitaire est axée davantage vers la théorie, bien que l'on retrouve de plus en plus de recherche appliquée dans de nombreuses disciplines. De plus, la documentation et la conservation des arts médiatiques avaient été peu investiguées de cette manière, jusqu'à maintenant, par les musées ou les universités du Canada.
M.-H. FoISY \& M.-È. COURCHESNE Selon vous, pourquoi existe-t-il à l'heure actuelle, dans la communauté muséale, un sentiment d'urgence pour l'élaboration et la mise en place de stratégies de conservation des cuvres à composantes technologiques?

\section{SyLvie Lacerte}

Parce que plusieurs collections de musées d'art et d'architecture, et non seulement dans les musées partenaires de DOCAM, sont aux prises avec un corpus d'œuvres utilisant des technologies qui datent des quarante dernières années et dont l'obsolescence fait en sorte qu'elles fonctionnent mal ou plus du tout. L'obsolescence des technologies est multifactorielle et mérite que l'on s'y attarde davantage afin de trouver des solutions qui assureront la pérennité des œuvres. La désuétude des supports (ou des appareils) comme les ordinateurs, les lecteurs VHS, les moniteurs vidéo, dont les pièces ou les appareils complets ne se retrouvent plus surle marché, ou encore la détérioration des médiums sur lesquels les œuvres sont enregistrées, tels les logiciels ou les bandes VHS, sont des exemples qui rendent l'entreprise urgente. En effet, les conditions de détérioration et l'instabilité des technologies en présence ont rendu plusieurs œuvres "illisibles" parce que les logiciels sont endommagés ou parce qu'on ne peut plus trouver d'appareils sur le marché pour en faire la lecture. Cela signifie qu'il pourrait être difficile, voire impossible, de réexposer ces œuvres si aucune mesure de conservation 
et de restauration n'était entreprise dans des delais rapides. Ajoutons à cela que les musées sont les "gardiens" de ce patrimoine pour le public qui contribue en grande partie au financement de leurs activités. La conservation, la recherche et l'exposition sont parmi les principaux mandats des musées. Il est donc important que la communauté muséale adopte des politiques et des protocoles pour assurer la préservation et la pérennité des œuvres. Et s'il est vrai qu'une partie de ce patrimoine est peutêtre est déjà condamnée à une date de péremption imminente, il n'est cependant jamais trop tard pour amorcer un projet comme DOCAM. D'autres projets similaires ont déjà vu le jour ailleurs dans le monde ou sont en voie d'être créés. Par exemple,aux États-unis, le projet Archiving the Avant-Garde de même que le Réseau des médias variables donneront lieu à une suite nommée Forging the Future. En Europe, le projet d'une durée de trois ans intitulé Inside Installations ${ }^{[3]}$, qui s'est terminé le printemps dernier, avait reçu son financement de la Commission européenne (CE).

\section{[3]}

<http://www.inside-installations.org>.

[4]

<http://www.tate.org.uk/conservation/time/about.htm>.

Voir aussi le projet Media Matters, un consortium incluant le Museum of Modern Arts (MoMA), le Tate Museum, le San Francisco Museum of Modern Art (SFMoMA) et le New Art Trust : <http://www.tate.org.uk/research/tateresearch/ majorprojects/mediamatters $>$.
Inside Installations par ailleurs comptait trente partenaires de la communauté muséale dans presque autant de pays. Par ailleurs, le Tate Modern à Londres s'est muni d'un département Time-based Media Conservation ${ }^{[4]}$, à l'instigation de sa directrice, la restauratrice Pip Laurenson, qui travaille avec des œuvres vidéo, des œuvres Web et des technologiques, de même qu'avec tout ce qui toucheà la performance et à la documentation de cette forme d'art; en définitive des œuvrespour lesquelles le temps (dans le sens de la durée) est l'une des données fondamentales. Naturellement, il est important de créer et de maintenir des liens avec les personnes qui sont au cœur de ces projets de recherche et avec les musées del'étranger, préoccupés par ces enjeux, afin d'échanger avec eux sur les préoccupations et les problématiques qui nous sont communes. Ces échanges sont féconds et riches d'enseignements, car plusieurs de nos interlocuteurs ont déjà amorcé la réflexion et ce travail. Par ailleurs, DOCAM a aussi entrepris certaines initiatives que les autres projets n'ont pas nécessairement planifié, tout dépendant du contexte, des budgets et des priorités de chacun. Les échanges sont donc essentiels, puisque ce réseau permet que nos actions respectives se complètent les unes avec les autres. 
M.-H. FoISY \& M.-̇̀. CoURChESNE Il est intéressant de constater que personne ne travaille de façon isolée, qu'il existe une concertation.

\section{SyLVIE LACERTE}

Tout à fait, et nous ne souhaitons pas travailler en vase clos. C'est un travail collaboratif. Par exemple, leprojet InsideInstallationsquicomptait trente musées européens dans ses rangs a énormément bénéficié du savoir-faire des spécialistes provenant de ses institutions partenaires. Détenant un budget imposant octroyé par la CE, de grandes sommes d'argent ont $p u$ être investies pour mener des projets jusqu'à l'étape ultime de la restauration des œuvres et de leur réexposition, ce qui n'est malheureusement pas possible pour DOCAM. L'important cependant, c'est que le travail en réseau permet une prise de conscience qui a des répercussions sur les musées européens, américains et maintenant canadiens. L'Alliance DOCAM a également initié des partenariats internationaux et ailleurs au Canada. Aussi, DOCAM pourra peut-être inciter l'elaboration d'un réseau universités/musées qui se poursuivra au-delà du projet de recherche ${ }^{(5)}$, afin que des liens durables se matérialisent entre ces deux mondes institutionnels dont le mandat respectif comporte une part importante de recherche. En tous les cas, je pense qu'il sera difficile de reveniren arrière après la findu projet DOCAM.

\section{[5]}

Le projet DOCAM se terminera à la fin de l'année 2009.

\section{M.-H. FoISY \& M.-È. COURCHESNE}

Quels sont, selon vous, les défis majeurs qu'il faudra relever afin d'arriver à obtenir un système de conservation efficace des ceuvres à composantes technologiques?

\section{SYLVIE LACERTE}

La formation, actuellement lacunaire, est l'un des défis majeurs à relever, notamment par la création de cursus pédagogiques alliant théories et pratiques autour de ces enjeux, dans les programmes de premier, deuxième et troisième cycles en histoire de l'art, en arts visuels et médiatiques, en études et pratiques des arts, en communications, en musique, en sciences de l'information et en muséologie. La formation est un enjeu de taille puisque nous accusons un certain retard dans plusieurs domaines par rapport à des établissements d'enseignement en Europe et aux États-Unis. Plusieurs muséologues se sentent parfois démunis, car ils ne possèdent pas ces nouvelles connaissances. Ils doivent aussi avoir de bonnes justifications pour défendre des augmentations de leurs budgets auprès de leur hiérarchie et il est donc crucial de mettre des mécanismes en place pour les aider à développer cet argumentaire. Je pense qu'il serait intéressant, pour ne pas dire impératif, de créer, dans un proche avenir, des programmes de formation continue pour les professionnels actifs dans le milieu muséal. Ce serait un grand atout, puisque ces professionnels ne partiront pas tous à la retraite bientôt! De cette manière, nombre de 
restaurateurs, de catalogueurs, d'archivistes des musées canadiens qui n'auraient pas participé à DOCAM bénéficieraient d'outils pour assurer une mise à jour de leurs connaissances, ce qui les aiderait à "mettre la main à la pâte». Pour résumer, la formation de la relève et le perfectionnement des muséologues, des professionnels et des enseignants ( $y$ compris les artistes, plusieurs d'entre eux étant professeurs) sont les principaux enjeux et défis à relever. Avec bien évidemment des budgets àl'avenant!

M.-H. FOISY \& M.-È. COURCHESNE Au volet de la formation, pouvez-vous expliquer le fonctionnement des séminaires DOCAM?

\section{SYLVIE LACERTE}

Le séminaire DOCAM est une introduction à la documentation et à la conservation du patrimoine des arts médiatiques et est ouvert aux étudiants de plusieurs disciplines des cycles supérieurs des quatre universités montréalaises. Le projet DOCAM a planifié un programme pour alterner ce séminaire entre l'Université McGill (hivers 2006, 2008) et l'UQÀM (hivers 2007, 2009). Pour l'instant, ces séminaires sont assurés par DOCAM, avec la collaboration des universités partenaires. Toutefois, nous souhaitons que ces corpus pédagogiques soient intégrés de façon permanente dans les universités participantes. L'an dernier, des étudiantes (des femmes seulement) des quatre universités, avec des parcours très diversifiés, se sont inscrites au séminaire DOCAM. Elles provenaient de disciplines comme la muséologie, les sciences de l'information, les communications, les arts visuels et médiatiques et les études et pratiques des arts. Il est intéressant de constater que le séminaire répond à des besoins dans toutes les sphères des arts, mais aussi à celles des communications et des sciences de l'information.

$\mathrm{Au}$ départ, ce cours d'introduction ne peut être trop spécialisé, puisque nous avons affaire justement à des étudiants qui ont des profils et des intérêts différents. Pendant les séminaires, nous recevons des artistes, des technologues et des chercheurs de DOCAM, à titre de conférenciers, pour présenter leur spécialisation, toujours autour des enjeux de DOCAM (historique des technologies, catalogage, restauration, archivage, dynamique de travail entre artiste et technologue, etc.). Les travaux requis pour le séminaire portent, entre autres, sur des études de cas théoriques, mais aussi sur des œuvres existantes contenues dans les collections de certains musées ou chez les artistes, ou en voie d'être acquises par les musées. Les étudiants sont donc ancrés dans la réalité et les travaux n'en restent pas à une simple vue de l'esprit. 
À la fin d'une des sessions, j'ai demandé aux étudiantes de compléter un sondage (à ne pas confondre avec l'évaluation des enseignements de l'Université) sur leur appréciation des contenus transmis au cours de la session et sur la manière de transmettre ces contenus. À vrai dire, bien que la majeure partie des commentaires aient été très positifs, certaines étudiantes ont émis le souhait de "mettre davantage la main à la pâte " en réalisant, par exemple, un stage auprès d'un restaurateur ou une résidence d'observation (participative) dans un atelier d'artiste. Ces remarques démontrent qu'il existe vraiment un besoin de formation sur le terrain. DOCAM souhaiterait créer une suite à ce séminaire, toujours dans le contexte $d u$ projet de recherche, par le biais duquel les étudiants qui ont suivi le cours d'introduction pourraient travailler avec des artistes, des restaurateurs, des catalogueurs ou des conservateurs d'exposition. Enfin, la réflexion - bien amorcée - sur ce deuxième cours " pratique " est encore au stade embryonnaire. Une particularité du séminaire DOCAM est que les budgets de recherche nous autorisent à recevoir des conférenciers à presque tous les cours ou à réaliser des sorties dans des laboratoires de restauration, comme cela s'est produit pendant la session d'hiver 2007. Nous sommes conscients que la réalité budgétaire universitaire est tout autre et que le programme ou le séminaire devrait adopter une autre forme.
Il est important d'être conscient qu'aucun professeur ne peut détenir la connaissance de toutes ces spécialisations. Néanmoins, je tiens à souligner que tous les chercheurs DOCAM qui ont été conférenciers depuis deux ans l'ont fait gracieusement. Cela fait partie de leur contribution au projet de recherche. DOCAM tient toutefois à rémunérer les artistes et les technologues qui ne sont pas attachés au projet de recherche.

Nous devrons donc réfléchir encore au fonctionnement dece séminaire. Celadit, même si les mécanismes des enseignements peuvent poser une certaine difficulté, il est essentiel que le séminaire devienne permanent. D'ailleurs, Louise Poissant, doyenne de la Faculté des arts à l'UQÀM, est à pied d'œuvre pour ouvrir un cours permanent dans un des programmes de la Faculté. Lorsque les étudiants des programmes en muséologie arriveront au terme de leurs études et qu'ils souhaiteront devenir catalogueurs ou conservateurs d'exposition, ils devront savoir comment échanger sur les contenus et les technologies, du moins de manière globale, avec les artistes, les technologues, parfois les programmeurs et les ingénieurs pendant le montage d'une exposition, par exemple, ou avant l'acquisition d'une œuvre. Cela représente une tout autre dynamique par rapport à des disciplines traditionnelles pour lesquelles les médiums sont plus stables. Cette dynamique et ces savoir-faire pourraient 
être transmis, $d u$ moins en partie, au cours d'un séminaire ou d'un programme court, lequel, à l'instar du séminaire DOCAM, exposerait les étudiants à cette réalité.

Actuellement, l'exposition E-art : nouvelles technologies et art contemporain - les dix ans d'activités de la fondation Daniel Langlois est en montage au MBAM. Ce sera la première fois à Montréal qu'une aussi vaste exposition de ce type, contenant le travail de dix artistes, présentera des ouvres strictement technologiques.Jean Gagnon, commissaire invité d'E-art, a notamment occupé le poste de conservateur en arts médiatiques au Musée des beaux-arts du Canada, mais, depuis son départ du musée il y a dix ans, le poste a été aboli. Il y a encore très peu de musées qui ont un poste de conservateur en arts médiatiques. Éventuellement, les musées devront faire appel à des conservateurs qui connaissent bien les œuvres technologiques et les artistes qui pratiquent ce genre de démarches, tant sur les plans historique et esthétique que technologique.

[6]

Voir le Glossaire du Réseau des médias variables : <http:// www.variablemedianet/pdf/Glossaire_FRA.pdf>.
M.-H. FoISY \& M.-È. COURCHESNE

Que pensez-vous des différentes solutions qui sont apportées aujourd'hui, telles que l'émulation, la migration et la réinterprétation, et de quelle manière croyez-vous qu'elles peuvent affecter l'authenticité des œuvres?

\section{SYLVIE LACERTE}

Cette question est très importante, même cruciale. D'ailleurs, l'Institut du Patrimoine et la Faculté des arts de l'UQÀM organisent conjointement une journée d'études le 23 novembre prochain, intitulée "Les enjeux de la conservation des œuvres fragiles, éphémères et virtuelles. Comment penser leur authenticité matérielle et conceptuelle?". Lorsque des modifications doivent être apportées à une œuvre incluse dans une collection muséale en raison de l'obsolescence des technologies, le musée doit naturellement obtenir l'autorisation de l'artiste avant d'entreprendre quel que processus que ce soit, qu'il s'agisse de migration ou d'émulation. Certains artistes sont d'accord avec ces modifications, d'autres pas. Plusieurs variables sont en cause dans cette décision: avec la migration, qui entraîne la mise à niveau des équipements, par exemple en changeant un moniteur de télévision des années 1980 pour un écran plat, on risque de changer l'effet de l'œuvre puisque les technologies ne sont plus les mêmes; avec l'émulation, on tente au contraire de conserver le même effet, d' "imiter l'apparence d'origine, avec des moyens tout à fait différents" ${ }^{[6]}$. Alors, tout dépend du musée 
et de l'ampleur des travaux que celui-ci est prêt à entamer et des sommes qu'il consentira à investir pour la restauration d'une œuvre, que ce soit par le biais de l'émulation ou de la migration, dans le but de réexposer. Quant à la réinterprétation, si vous avez en tête la démarche que Marina Abramovic a présentée au public en 2005 au musée Guggenheim de New York, avec sa série de Re-enactments, Seven Easy Pieces, DOCAM ne s'est pas vraiment penché sur la question. En revanche, une de nos assistantes de recherche, doctorante en histoire de l'art à McGill, a écrit un article qui paraîtra dans le numéro d'automne 2007 de la revue Leonardo sur la démarche d'Abramovic et les problèmes posés par la documentation de cet événement. Mais il est vrai que cette question pourrait poser certains problèmes éthiques, par rapport à l'authenticité de l'œuvre originelle. Il faut se poser des questions sur l'aspiration des artistes ou des musées à l'immortalité d'une œuvre. Le musée dont le mandat premier est de conserver le patrimoine pour l'éternité ou, à tout le moins, le plus longtemps possible au meilleur de ses capacités et de ses limites, doit savoir si les artistes souhaitent queleurs œuvres soient pérennes. Carle musée doit être en mesure de suggérer des solutions pourla conservation des œuvres, mêmesi, inévitablement, certaines ne pourront pas être conservées éternellement. Lors du Sommet DOCAM 2006, Pip Laurenson relatait la démarche de la Tate auprès des artistes pour leur démontrer que les restaurateurs tentaient deprolongerlavied'uneœuvre, avecles moyens actuels à leur disposition, mais qu'il était plus sage d'être conscient du fait que certaines œuvres, connaissant les technologies en présence, auraient une date de fin de vie $\mathrm{X}$ ou de péremption, au terme de laquelle l'œuvre n'existerait plus. Cela signifie que des cuvres médiatiques ou technologiques pourraient avoir, par exemple, des durées de vie limitées de 10, 20, 30, 40 ou encore 100 ans. Il est donc important d'établir des protocoles pour la restauration et la préservation des œuvres et la réexposition. Il faut parfois savoir tirer la ligne et ne pas faire preuve d' "acharnement thérapeutique" sur les œuvres pour assurer leur survie coûte que coûte, puisque ces actions pourraient justement entacher l'authenticité même de l'œuvre. Par contre, il est clair, dans l'intérêt de la préservation du patrimoine des arts médiatiques, que la communauté muséale doit tenter de garantir la survie d'une œuvre en s'assurant de conserver son authenticité et son intégrité. Mais il faut peut-être se résigner à l'impossibilité de réaliser cette tâche, dans tous les cas de figure, soit parce que les moyens techniques ne nous le permettront pas, soit simplement parce que l'artiste ne le souhaite pas. 
Comme partie du projet des Médias variables, l'exposition Seeing Double ${ }^{[7]} a$ été réalisée et présentée au musée Guggenheim à New York. Il y était justement question d'émulation-avec, notamment, les œuvres The Erl King, 1982-1985, de Grahame Weinbren et Roberta Friedman - et de migration - avec T.V. Crown, 1965, de Nam June Paik. Dans le projet DOCAM, il y a par ailleurs une étude de cas en cours, au sein du comité Structure de catalogage, sur une œuvre de Paik de la collection du MBAM. Cette œuvre acquise par don au debut des années 1990 n'a jamais été exposée au public, parce qu'elle ne fonctionnait pas au moment de son entrée dans la collection du Musée (en raison d'eléments défectueux). DOCAM aide concrètement le MBAM, puisque l'étude de cas permettra aux professionnels de cetteinstitution d'établir un protocole pour le catalogage et ainsi trouver, éventuellement, des solutions pour la restauration de l'œuvre. S'il devenait inévitable d'entreprendre une migration, en remplaçant les composants originels pour être en mesure de continuer à faire fonctionner l'œuvre, et advenant que les composants de remplacement soient introuvables, il faudrait alors obtenir l'autorisation de la succession de Paik.

[7]

$<$ http://www.variablemedianet/f/seeingdouble/index. htm $>$.
Il est vrai que les œuvres n'ont pas été créées pour demeurer dans les réserves éternellement et il est parfois difficile de les garder en vie et fonctionnelles pour l'éternité!

$$
\text { M.-H. FOISY \& M.-È. COURCHESNE }
$$

De quelle manière croyez-vous que les cuvres à composantes technologiques et tous les nouveaux enjeux de conservation qui en découlent influenceront le milieu de la muséologie?

\section{SYLVIE LACERTE}

La présence des cuvres d'art médiatiques dans le patrimoine muséal et les enjeux qui en découlent auront des conséquences sur les manières de pratiquer la muséologie. Et l'une des solutions à ce changement de paradigme passe, entre autres, par une formation renouvelée qui tient compte de ce contexte. Si DOCAM apporte, ne serait-ce qu'une partie des solutions, en offrant des outils comme des guides de bonnes pratiques en catalogage et en conservation/ restauration, cela encouragera possiblement les musées à acquérir des œuvres médiatiques. DOCAM souhaite aussi fournir àla communautémuséale des pistes concernant le travail à effectuer pour la documentation, la terminologie et la réexposition des cuvres. Je pense aussi que cela incitera encore davantage les universités à créer des nouveaux cursus pour former des muséologues qui deviendront, en conséquence, plus aguerris à ces nouvelles pratiques. L'Alliance DOCAM est d'avis qu'il est vraiment important d'aller dans cette 
direction, puisque ces pratiques n'iront pas en diminuant. Cela ne signifie pas non plus que tous les artistes auront des pratiques médiatiques. Il $y$ aura toujours des artistes dont la démarche fera moins appel aux technologies (dans le sens des nouveaux médias). On prendra conscience aussi que la formation de la relève et celle des muséologues actifs dans le milieu est primordiale.

M.-H. FoISY \& M.-È. COURCHESNE

Que pensez-vous de la formation offerte au Québec en ce qui a trait à la conservation des œuvres à composantes technologiques?

\section{SYLVIE LACERTE}

J'ai déjà un peu répondu à cette question et ne voudrais pas m'éterniser sur le sujet, mais disons que la formation en restauration d'œuvres d'art n'existe pas encore au Québec. Un programme de maîtrise est offert à la Queen's University de Kingston, Ontario. Seulement, les professeurs ne sont pas formés pour enseigner ces nouvelles pratiques de restauration et de conservation. Le programme Art Conservation de Queen's est l'un des partenaires de DOCAM et les étudiants viennent au Sommet annuellement. Malheureusement, ils ne peuvent assister au séminaire, surtout en raison de la distance qui sépare Montréal de Kingston, mais aussi parce que le programme très intensif exigerait des étudiants qu'ils se rendent à Montréal sur une base hebdomadaire.
Malgré les lacunes de la formation québécoise, les assistanats de recherche de DOCAM constituent une bonne école. Une étudiante de Queen's a travaillé sur le fonds Vera Frenkel aux archives de l'Université Queen's. Vera Frenkel est une artiste multidisciplinaire qui travaille beaucoup avec les technologies et le Web. Une autre étudiante, qui a été assistante de recherche pour le Séminaire DOCAM 2007, assiste actuellement Jean Gagnon pour l'exposition E-art : nouvelles technologies et art contemporain, comme partie de son stage de maîtrise en muséologie. Une doctorante en études et pratiques des arts a été assistante de recherche au MBAC (au laboratoire de restauration de Richard Gagnier $\left.{ }^{[8]}\right)$ et a travaillé sur des études de cas d'œuvres de Stan Douglas (Nut'ka) et de David Rokeby (Machine for Taking Time). Une autre étudiante à la maîtrise en muséologie réalisera son travail dirigé sur DOCAM. Une stagiaire, qui a aussi été étudiante en muséologie, aide à l'organisation $d u$ Sommet 2007 et plusieurs autres assistanats de recherche sont en cours.

\section{[8]}

Richard Gagnier est président du comité conservation/ restauration. Il a été restaurateur de l'art contemporain au MBAC pendant plusieurs années et, depuis septembre 2007, il est restaurateur en chef au MBAM . 
Parle biais des assistances de recherches, DOCAM offre en quelque sorte une formation pratique. Ce travail sur le terrain et les assistanats de recherche sont en général grandement appréciés par les professionnels des musées partenaires de l'Alliance DOCAM. Le transfert des connaissances se fait dansles deux sens, puisque certains des assistants de recherche, férus de technologie, ont la possibilité de transmettre des savoir-faire aux conservateurs, aux restaurateurs ou aux catalogueurs, plus habitués à des supports et à des manières de faire traditionnels. Les échanges entre les muséologues et les assistants de recherche sont donc particulièrement féconds. De plus, ce travail donnera lieu à des publications, autant sur le site Web de DOCAM que dans des revues savantes, des magazines professionnels et des revues d'art. C'est vraiment intéressant de voir tout ce que cela peut créer. Il est vrai que c'est un processus qui prend un certain temps, mais disons que nous en consolidons actuellement les bases en élaborant des nouveaux savoir-faire et des formations. Cette année, DOCAM a créé un comité des assistants de recherche, par le biais duquel ceux-ci se réunissent quatre à cinq fois l'an. De plus, un représentant des assistants siège au comité de direction de l'Alliance. Ces réunions du comitédes assistants de recherche ne sont pas strictement administratives. Au contraire, ce sont des rencontres où les assistants présentent l'évolution de leurs travaux. Il y a vraiment une émulation qui se transmet entre les étudiants eux-mêmes, afin de partager leurs réflexions et leurs recherches. Ce comité est une véritable tribune d'échanges qui s'ajoute au blogue des chercheurs auquel les étudiants contribuent de façon régulière.

Dans un autre ordre d'idées, par le biais du site Internet de DOCAM, la communauté a accès aux syllabus des deux derniers séminaires DOCAM, aux lectures recommandées et à certaines des conférences qui y ont été présentées, grâce à des captations vidéo, mais aussi à des capsules produites par les étudiants pour la veille thématique de DOCAM. Nous tentons le plus possible d'être ouverts aux communautés universitaires (étudiants et professeurs), artistiques et muséales, tout en réalisant un type d'e-learning qui représente un autre genre de formation. Tout cela nourrit les contenus de notre site Internet et nous en sommes très fiers. 
M.-H. FOISY \& M.-È. COURCHESNE

Quelle place l'Alliance DOCAM occupe-t-elle dans ces grands enjeux ? Où en est-elle dans sa réflexion et quelles actions pose-t-elle en ce moment sur ses propres collections?

\section{SYLVIE LACERTE}

DOCAM en soi ne possède pas de collection, puisque c'est une Alliance de recherche. Ce sont les musées partenaires qui détiennent les collections. En fait, pour faire la synthèse des objectifs de DOCAM, ce qui a intéressé tous ses partenaires muséaux et universitaires a été de "mettre la main à la pâte" dans un travail de collaboration pour développer des outils en établissant des protocoles d'action ou en rédigeant des guides de bonnes pratiques sur les mesures à prendre en documentation, en catalogage, en conservation / restauration, pour une meilleure préservation des œuvres, donc de conscientiser la communauté muséale. Le Réseau canadien de l'information sur le patrimoine est l'un de nos partenaires; il nous offre un soutien inestimable par des subventions pour des postes de stagiaires et il offrira à DOCAM une tribune pour la diffusion des résultats de recherches, etc., tout cela pour mettre une panoplie d'outils à la disposition de la communauté muséale. En outre, le sommet annuel est un important canal de diffusion qui permet aux chercheurs de livrer des données sur l'évolution de la recherche à une bonne partie de la communauté. En définitive, l'un des objectifs principaux de DOCAM est de conscientiser la communauté à l'importance des enjeux et des défis que nous aurons à relever dans les années à venir.

M.-H. FoISY \& M.-È. COURCHESNE Comment entrevoyez-vous l'avenir des collections des musées et celui du rôle de leur conservateur en ce qui concerne les ceuvres à composantes technologiques?

\section{SYLVIE LACERTE}

Je perçois l'avenir d'une façon assez positive. Par exemple, au Centre Canadien d'Architecture, nous travaillons avec des restaurateurs et des conservateursd'exposition quin'avaient utilisé que le papier comme médium principal de leur merveilleuse collection. Maintenant, ils sont en voie d'acquérir des fichiers numériques. Pour cesprofessionnels, l'univers du numériqueétait totalement étranger. Mais au regard des nouvelles acquisitions et avec l'aide de DOCAM, ils ont dû $y$ plonger avec, il est vrai, une certaine appréhension au départ. Grâce aux assistants de recherche très au fait des technologies et de l'architecture virtuelle, ils ont découvert un monde tout à fait extraordinaire qui leur a permis d'aller chercher des pistes de solutions qu'ils n'auraient pas envisagées auparavant. C'est ce qui est vraiment fascinant dans ce projet de recherche. Ils ont saisi cette nouvelle réalité et ne travaillent, d'ores et déjà, plus de la même façon. Le CCA a même crééàl'intérieur de son musée un groupe de travail qui se penche spécifiquement sur les problématiques et les enjeux des dossiers et des œuvres numériques. 
Nous sommes très heureux de constater que l'Alliance DOCAM a suscité une amorce de changement dans la culture des institutions. Changer les façons de faire est ce qui prend le plus de temps à réaliser, autant à l'intérieur d'un musée que d'une université. Mais je maintiens que nous sommes sur la bonne voie.

Ce qui serait vraiment fantastique, c'est qu'un séminaire DOCAM numéro deux soit créé. En cinq ans, un projet de recherche aussi vaste que celui-ci ne nous donnera peutêtre pas le temps d'aller jusqu'au bout de la démarche. Les initiateurs de DOCAM, Jean Gagnon et Alain Depocas, n'avaient pas non plus imaginé l'ampleur que prendrait ce projet. Il est illusoire de croire que nous pouvons trouver les solutions à tous les maux en cinq années seulement, que nous pouvons assister à la transformation de toutes les façons de faire et à toutes les cultures institutionnelles. L'important demeure néanmoins d'avoir jeté les bases d'une nouvelle manière de travailler et d'une nouvelle façon de réfléchir à ces enjeux.
M.-H. FOISY \& M.-È. COURCHESNE Et poursuivre la concertation avec les institutions européennes et états-uniennes?

\section{SYLVIE LACERTE}

Absolument. La collaboration entre institutions est vraiment importante. Elle est synonyme de changement de culture. Il est sûr que les institutions ne peuvent pas partager tous leurs "secrets d'État", ce qui est tout à fait légitime. Toutefois, l'union fait la force et, lorsque nous sommes en mesure de partager et d'échanger sur des problématiques communes, il est plus facile de trouver des solutions. Nous avons constaté avec DOCAM, et les autres projets comme Médias variables, Media Matters, Archiving the Avant-Garde et Inside installations, que l'union fait vraiment la force. Le partage et le réseautage sont essentiels au succès de ces entreprises et à la pérennisation du patrimoine des arts médiatiques; ils permettent d'offrir aux musées des outils qui les aideront à poursuivre leur mandat de bons gardiens de tous les patrimoines, autant antiques que ceux des nouveaux médias. 